\title{
Visualization of Storm Surge based on GIS
}

\section{Xifang $\mathrm{JIN}^{1, \mathrm{a}}$, Fangzheng WANG ${ }^{2, \mathrm{~b}}, \mathrm{Lili}_{\mathrm{CHEN}}{ }^{2, \mathrm{~b}},{\mathrm{Jie} \mathrm{LI}^{2, \mathrm{~b}}, \text { Wei ZHAO }}^{2, \mathrm{~b}}$}

${ }^{1}$ North China Sea Marine Forecasting Center of Station Oceanic Administration,North

China Sea Branch of State Oceanic Administration, Qingdao, 266061,China

${ }^{2}$ College Of Geomatics,Shandong University of Science and Technology,Qingdao,266590,China

aemail:419958059@qq.com, bemail:407615037@qq.com

Keywords: Storm Surge; visualization; display control; dynamic expression

\begin{abstract}
The core of this paper is the visualization of the multi-source data of the storm surge. The multi-source data includes the flow-field, water level, sea temperature, wave, wind-field and some other prediction factors. Around the visualization, some creative work has been done in this paper. Firstly, for the large amount and high density dynamic field data such as the flow field, wave direction, the wind-field, etc. In this paper, a uniform discretization algorithm is designed according to the elements of its characters. On one hand, it controls the display range of the factors. On the other hand, it controls the scale calibration. This algorithm is to make the power-field data to zoom steplessly. Secondly, as the storm surge data are almost long-time series data, this paper form the perspective of different design with two different solutions in order to better reflect the variation of storm surge with time, and the dynamic visualization of the prediction factors within a certain time sequence are realized.
\end{abstract}

\section{Introduction}

The work of storm surge forecast started in the 1920s and 1930s. Early storm surge prediction mainly depends on the method of experience statistical forecast including the forecasters' subjective experience and the method of experience statistical forecast [1]. Numerical forecast began to appear until 1950s. The development of numerical prediction is very rapid, after twenty-first Century, numerical prediction has been the main forecasting means of storm surge [2]. Numerical prediction has the advantages of less test cost, fast speed, short cycle and so on, but it focuses on the theoretical and numerical models for parametric studies. At the same time, the numerical prediction results are a lot of boring data and a variety of still static images what base on the data produced. The display is not intuitive, and does not reflect the vivid image of the hydrodynamic phenomenon, while the spatio-temporal data of storm surge has the characteristics of dynamic, fuzziness, spatiotemporal process and temporal granularity [3]. Therefore, "establish precise dynamic display system of simulation graphics" is one of the directions of development of the numerical prediction [4].

After entering the twenty-first century, storm surge studies tend to be more refined. In 2004, Hongcai Li et al. used Fortran Power Station4.0 and Visual Basic6.0 as the numerical calculation language and the graphical developing software to design visualization of Guangdong coastal tidal prediction system of typhoon storm [5].In 2007,Jianzhong Ge developed a storm surge forecasting integrated visualization system with VB, Fortran and Matlab [6]. These studies expressed a large number of data of storm surge as various static and dynamic images, which can better realize the storm surge numerical prediction system integration. But they didn't rarefy processing of massive data, which made the data show complex, low efficiency, and can't execute editing operation the map related in the animation process. To solve the above problems, based on the reference of other visual prediction system, this paper adopts the prediction of storm surge and GIS integration technique, thinning processing elements of the storm surge forecast in space, so that the display efficiency of the massive field data is improved, and dynamically demonstrating the forecast data with two scheme, which can not only increase the speed of the dynamic demonstration but also execute reoperation with the data. 


\section{The Data Types of Forecast Factors}

Forecast factor is the Information Center of the storm surge Visualization, including: flow field, water level, sea surface temperature, wind field, wave, etc, what are spatial data products. As the data is the feature of dynamic, fuzziness and multi-source, when it comes to organize and manage the original data (NETCDF format and binary storage format), it is necessary to analyze the data according to its characteristic, and convert it into the data formats that GIS platforms support, so that the difficult problem of data input is solved.

(1). Flow field, wind field and wave direction, which are the vector field data, are respectively converted into Shapefile or Personal Geodatabase, and special symbols are used (such as the arrow symbol, the wind vector symbol) when rendering.

(2). Sea temperature, water level, wave height, which are the grid data, are respectively converted into TIN or RASTER, color grading is only required when rendering.

\section{The Sparse Display of Massive Field Data in the Space}

\section{Problem analysis}

The massive field data (flow field, wind direction, wave direction) has characteristics of large amounts of data and display elements of many. If the data is full of display, it would be very dense, has a poor display effect and low display efficiency, such as flow field, shown in Figure1 (a), the image is flow field of numerical simulation in the area of Qingdao Jiaozhou Bay, a total of about 34000 data points. When it is full of display, data overlap each other, and it is unable to see the overall trend and detail information of the flow field.

In order to solve map display problems containing large amounts of intensive data, this paper is based on GIS space display control technology, and realizes the data quasi zooming in different scale, which ensures the accurate display of vector field distribution and variation characteristics. After processing, the display effect of flow field shown as Figure1(b), amount of the flow field data points is only retained about 3000, moderately spaced moderate interval, and the feature of the whole flow trend is retained well.

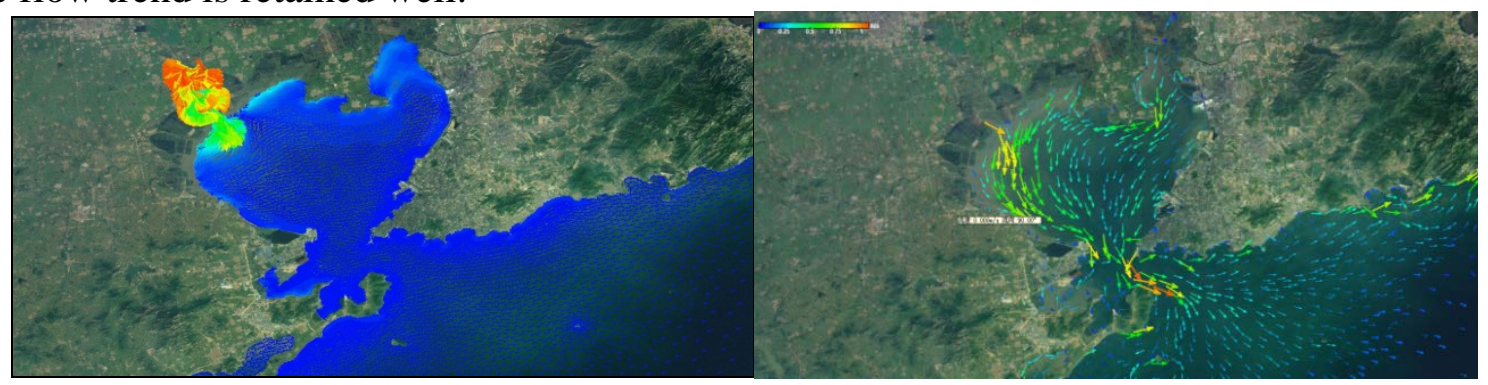

(a) all flow field elements

(b)flow field elements after display control

Fig.1. Effect drawings of the flow field

\section{The solution}

This paper mainly realizes the uniform display data from the following aspects:

\section{(1) Dynamic adjustment of the display range}

Range control of the data display is refers to extract the display information by coordinate information of the vector field data, the vector field data will only be displayed within the scope of the screen, vector field data outside the range of the screen will not be displayed, the problem of a large amount of data and low display efficiency can be solved.

(2) sparseness of Intensive vector field data

This paper adds ratio calibration control for the display elements, and a corresponding set of data are respectively extracted for each scale level that can be displayed on the scale to ensure the uniform and moderate interval. So when data is browsed, the amount of spatial data displayed in GIS will automatically increase or decrease with the change of the scale. But the change of the scale is a continuous process, and so much calculation is need in order to realize the stepless zoom for all 
scales that burden of computer is too big, what is not possible to implement. Therefore, the only way is to use stepped scales to simulate the stepless zoom.

\section{Technical process}

Vector field data can be divided into two categories according to the way of storage, the regular grid and irregular grid. Regular grid data are mainly the data such as wind data and wave data, and irregular grid is the flow field data. Storm surge system designs two sets of display control scheme respectively from quasi-stepless zoom in the different scales and the data range control of display according to the two kinds of data storage format.

(1) Sparseness and display of the flow field

A. The data step generation .The scheme is the data sparseness based on the virtual grid. It is to adopt rules grid to cover the entire vector field data, and each grid cell extract no more than a display element, so that a set of data is obtained. For the different scale section, setting the different grid size can gain display data of any scale, getting the segmented data sets in all intervals of display, among what the cell size controls the degree of sparseness. Generally the grid cell size is not less than the average distance between data points. The greater the grid cell size is the more data points need to remove; instead, the less data points need to remove.

B. The display control of flow field.Three fields are added to the flow field data during the previous processing of the scheme, including scamin, $\mathrm{X}$ and $\mathrm{Y}$. The scamin field value is denominator of the scale, and can be set according to the different levels of regular grid. The $\mathrm{X}$ field value is $\mathrm{X}$ coordinates of the vector points and $\mathrm{Y}$ field value is $\mathrm{Y}$ coordinates of the vector points. Scamin field is used to control the visibility of the vector points at the current scales. $\mathrm{X}$ and $\mathrm{Y}$ fields are used to judge whether the vector points is within the scope of the current screen or not, and the flow field data is visible when it is true, and vice versa. The SQL statement for the display control of flow field is shown following:

$$
p S Q L=x \epsilon(x \min , x \max ) \& \& y \epsilon(y \min , y \max ) \& \& \text { scamin }>\text { mapScale }
$$

Among this: xmin and xmax are the maxmum value and minimum value of $\mathrm{X}$ coordinate of the scope of the current screen, and ymin and ymax are the maxmum value and minimum value of $\mathrm{Y}$ coordinate of the scope of the current screen. mapScale is denominator of the current scale.

(2) The sparseness and display of wave direction and wind field

A. The data step generation .The scheme is based on the sparseness of system. Firstly, the row and column number of each point need to be defined according to the coordinates of data points. Secondly, setting the dilute factor according to the needs of users, the convenience of data processing and the aesthetic property of display, that is to say that it keep one point every $\mathrm{N}$ point. The advantage of using these two methods is that the algorithm processing is so quick and the algorithm is easy to understand.

B. The display control of flow field.Two fields, $\mathrm{i}$ and $\mathrm{j}$, are added to the wind field and wave data, during the previous processing of the scheme, what are the row number and column number of the vector point .The position, number and interval of the wind field and the wave vector data points are fixed, so the $\mathrm{i}$ and $\mathrm{j}$ field value of vector points is also changeless. Related calculation is executed according to the $\mathrm{i}$ and $\mathrm{j}$ field value, so that the visibility of each vector point can be controlled at the current scale and within the scope of the current screen. The SQL statement for the display control of wave direction and wind field:

$$
p S Q L=i \epsilon(\text { imin, imax }) \& \& j \epsilon(\text { jmin, jmax }) \& \& s c a m i n>\text { mapScale }
$$

(2)

Among this:

levelInteval $\epsilon(1,20)$

(3)

$$
\text { imin }=(\text { Xmin }-y \min ) / \text { interval }
$$

(4)

imax $=($ Xmax $-y \min ) /$ interval 
(5)

$$
\text { jmin }=(\text { Ymin }-x \min ) / \text { interval }
$$

(6)

$$
\text { jmax }=(\text { Ymax }-x \min ) / \text { interval }
$$

(7)

Xmin and Xmax are the maxmum value and minimum value of $X$ coordinates of the scope of the current screen;Ymin and Ymax are the maxmum value and minimum value of Y coordinates of the scope of the current screen;xmin and ymin are the minimum latitude value and the minimum longitude value of wind field and wave direction data;interval is the interval among the vector points.

20 interval levels, from MapScaleMin to MapScaleMax, are setted by the distribution test of vector dot density of the wind field and wave direction, and Judge the range of the current screen scale (MapScale) denominator , and determine levelInteval values.

\section{Dynamic Expression at the Time}

The storm surge data are mostly long time series data with dynamic. In order to reflect the change trend of storm surge data better, two different schemes are designed from the different angle to realize the dynamic visualization of forecast factors expression in certain time series range. Simulate the dynamic changes of data over time based on the time attribute of field data, and make the display of field data changes over time more vivid and accurate.

(1) Multi-layer control display function based on GIS

During the process of data preprocessing, field data of storm surge forecast factors in different time are resolved as the layer file. The scheme is based on the Multi-layer display control of GIS, and realizes the dynamic play of storm surge forecast factors by controlling the visibility of each layer. Its disadvantage is that the reaction speed of vector field data is slow and the dynamic playback is not fast with limited to about 1 second and showing missing individual layer problem will appear if playback speed is greater than 1 second because of the refresh mechanism of GIS layer ; its advantage is in process of dynamic prediction of forecast factors, map can be enlarged, reduced, mobile and etc., which is convenient for forecasters to know more detailed information

(2) Mapping output function based on GIS

Previous processing is the first step in the schemes, which means mapping output the region that dynamic expression is needed, generating a series of pictures (in different time period) and then to control the play of these pictures to achieve the dynamic prediction factor expression. Its disadvantage is that related map operation cannot be executed, and need some processing time; its advantage is that the problem of slow playback speed of vector field data is solved.

According to advantages and disadvantages of the above two schemes, storm surge system takes some measures to integrate remedy. On the one hand, the design of a map label function makes forecasters complete the process of mapping output pretreatment in advance, save the map label, and view through the drawing label directly without preprocess; On the other hand, the layer control is associated with map label based on above two schemes, so that we can switch two programs at any time in the process of the dynamic forecast factors play, showing the same effect of forecast factors. It not only can solve the problem of slow vector data playback, and can reoperation on data in the process of dynamic play

\section{Results}

It is the highlight and innovation of the storm surge system that spatial and temporal expression of forecast factors were dynamically played in time and display controlled in space. While GIS has the function of visual expression of information, multi-source spatial information management, geospatial analysis and so on, making it the ideal platform for visualization information system of storm surge. ArcGIS, developed by American ESRI, is the professional software platform of GIS field that has comprehensive functions, widely usage and representativeness in the application. 
In this paper, where ArcGIS is used as the prototype development platform, realizes the dynamic visualization and the homogeneous sparse in spatially of the storm surge prediction data. This paper displays the effect in the control of space and dynamical play in time, shown with 3 pictures based on flow field as example.

(1) Display Control in Space

The space display control of forecast factor is to sparse display elements in different scale of display elements, which does not affect the accurate expression of forecast factors, but also beautify the display effect. Figure 2 (a), (b) and (c) are renderings of the flow field on the same area in different scales. It can be seen that, with the enlargement of the scale, display elements increase gradually as well, especially in the river.

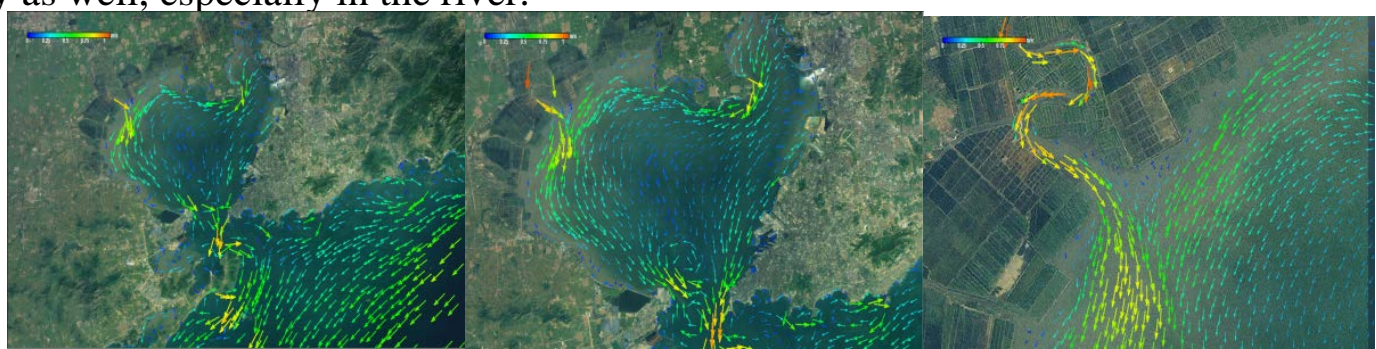
(a) in small scale
(b) in middle scale
(c) in large scale

Fig.2. Flow field display in three scales

(2) Dynamic Play in Time

Figure 3 (a) , (b) and (c) are the renderings of the flow field at the 23rd,24th,25th hour, that play control toolbar is being used to dynamically play. The change trend of two vortexes can be obviously seen when the three figures are observed together. Showing the dynamic changes of the flow field.

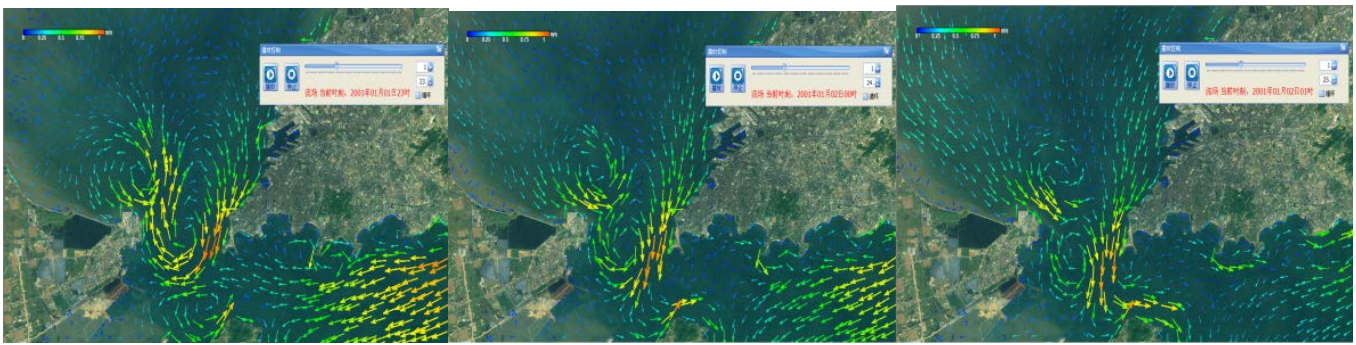

(a) at 23th hour

(b) at 24th hour

(c) at 25th hour

Fig.3. Flow field at three hours

Figure 4 (a) , (b) and (c) are the renderings of the wave height at the 23rd,24th,25th hour. It can be obviously seen that the wave height at different times are dynamically changing.

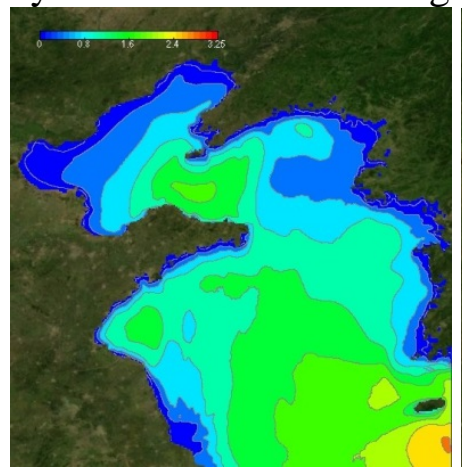

(a) at 23th hour

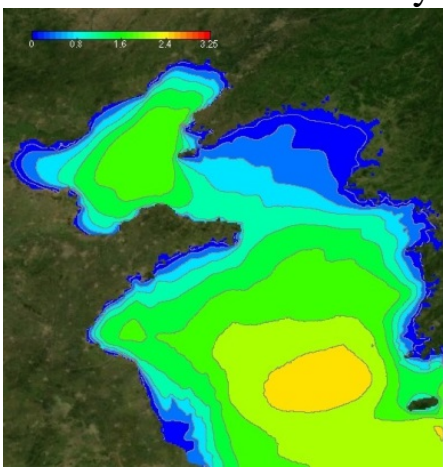

(b) at 24th hour

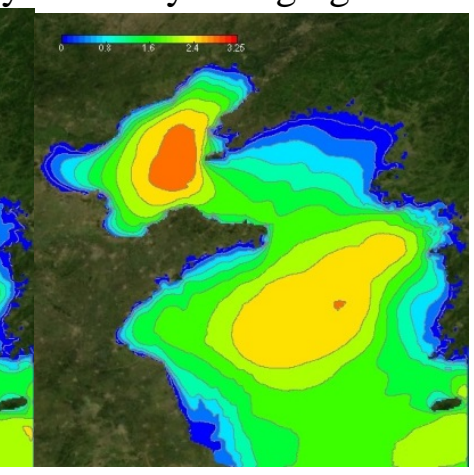

(c) at 25th hour

Fig.4. wave height at three hours

\section{Summary}

This paper provides a good platform for the visual expression of the storm surge forecast data with by integration technology of storm surge forecast and GIS, which can not only efficiently resolve and import forecast data, but also pay much attention to the study on the visual expression 
of the storm surge forecast data. It achieves the quasi-stepless zoom of ocean dynamic field data at different scales and solves the problem of poor display effect and low display efficiency. At the same time, in order to dynamically display the distribution and change of flow field, water level, wave height flow field, water level, given the distribution image and animation effects of flow field, water level, wave height at Gulf of Jiaozhou, this paper integrates two kinds of scheme, based on the multi-layer overlay display function and map output function of ArcGIS respectively. This paper solves the problem of the low speed of storm surge forecast factor dynamical expression, and can execute re-operation to the forecast factors during the process of dynamic visualization.

\section{Acknowledgment}

This paper are supported by Public science and technology research funds projects of ocean of which project number is 201205010-4, and of which project number is 201105016-4. We would like to express appreciations to colleagues in our laboratory for their valuable comments and other helps.

\section{References}

[1] Xi-nian Wang. Storm Surge Disaster and its Prediction and Defensive Countermeasures [J], MARINE FORECASTS, 1998,15(3):26-31

[2] Technical guide for prediction of storm surge [R] .NATIONAL MARINE ENVIRONMENTAL FORECASTING CENTER,2009:14-46

[3] Meizhen Zhao. The Research of ECDIS/Marine Spatio-Temporal Data Integration Technique [D].HARBIN INSTITUTE of TECHNOLOGY,2010:6-80

[4] Zude Cao, Yunhong Wang. Numerical Simulation of Hydrodynamics and Sediment [M] . TIANJIN:TIANJIN UNIVERSITY PRESS,1994

[5] Hongcai Li, Yongxin Wang, Shaoyi LIN. VISIBLE FORECASTING SYSTEM ON STORM SURGE ALONG

[6] GUANG DONG COAST [J]. marine forecasts, 2004,21(4):82-87

[7] Jianzhong Ge, Kelin Hu, Pingxing Ding. Design and Application of Integrated Visualized Storm Surge Forecasting System [J].JOURNAL of EAST CHINA NORMAL UNIVERSITY （NATURAL SCIENCES）,2007,(4):20-25

[8] Xinian Wang, Ye Lin. MONITORING AND FORECASTING OF STORM SURGES ALONG THE COASTAL AREAS OF CHINA [J]. MARINE FORECASTS,1993,9(3):28-37

[9] Junhua Teng, Wei Wu, Meixian Sun, Dongling LI, Fujiang YU. Aided decision-making information system based on GIS for disaster mitigation of storm surge [J].Journal of Natural Disasters,2007,16(2):17-21

[10] Huiying Gao, Shanjun Mo, Tian'en Chen. Research on emergency information management system of offshore disasters in Qingdao[J]. Journal of Natural Disasters, 2004, 13(4): 88-92. 